Rev. Elet. em Gestão, Educação e Tecnologia Ambiental (e-ISSN: 2236-1170)

\title{
AVALIAÇÃO DA QUALIDADE DAS ÁGUAS SUPERFICIAIS DO RIO JAMARI NA REGIÃO DA CONSTRUÇÃO DE UMA PCH NO MUNICÍPIO DE MONTE NEGRO-RONDÔNIA, AMAZÔNIA OCIDENTAL
}

\author{
Renato André Zan ${ }^{1}$, Ana Paula Araújo André2 ${ }^{2}$ Ana Paula Martins Coutinho ${ }^{3}$, \\ Nathália Vieira Barbosa ${ }^{4}$, Filomena Maria Minetto Brondani ${ }^{5}$, \\ Dionatas Ulises de Oliveira Meneguetti ${ }^{6}$ \\ ${ }^{1}$ renato-zan@hotmail.com, Químico, Mestre em Química, Docente e Coordenador de Pesquisa e Iniciação Científica \\ da (FAEMA), Faculdade de Educação e Meio Ambiente \\ 2 anapaula andre@hotmail.com, Graduanda em Licenciatura em Química da Faculdade de Educação e Meio \\ Ambiente (FAEMA) \\ ${ }^{3}$ anapcoutinho@hotmail.com, Graduada em Licenciatura em Química, Pós-graduanda em Ensino de Ciências e \\ Matemática da Faculdade de Educação e Meio Ambiente (FAEMA) \\ ${ }^{4}$ nathzinhavieira@hotmail.com, Graduada em Química, Mestre em Química, Docente e Coordenadora de Pós \\ Graduação da Faculdade de Educação e Meio Ambiente (FAEMA). \\ ${ }^{5}$ filomenabrondani@hotmail.com, Graduada em Química, Mestre em Biologia Experimental, Docente e Coordenadora \\ do Curso de Licenciatura em Química da Faculdade de Educação e Meio Ambiente (FAEMA). \\ ${ }^{6}$ dionatasmeneguetti@hotmail.com, Biólogo, Mestre em Genética e Toxicologia Aplicada, Docente e Coordenador de \\ Extensão da (FAEMA), Faculdade de Educação e Meio Ambiente
}

http://dx.doi.org/10.5902/223611707295

\section{RESUMO}

Com a construção de uma PCH (pequenas centrais hidrelétricas) no curso do Rio Jamari na cidade de Monte Negro - RO torna-se importante o monitoramento das águas superficiais do Rio Jamari, para avaliar sua atual situação durante o processo de implantação da $\mathrm{PCH}$, para posteriormente avaliar-se o impacto causado ao rio pela construção. Neste intuito o presente artigo trata da avaliação da qualidade da água do Rio Jamari, avaliando-se padrões físico-químicos, microbiológicos, fitoplâncton e ictioplâncton, onde após avaliar todos os parâmetros gerou-se o IQA (Índice da Qualidade de Água), que ficou entre 68 a 79\%, nos quatro pontos avaliados no curso do Rio, indicando uma água com qualidade boa, dentro dos parâmetros avaliáveis do IQA. Os valores obtidos foram considerados satisfatórios, tendo em vista que o Rio Jamari é um rio que sofreu muito com os processos de desmatamento da região, garimpos e implantação de lavouras as margens.

Palavras-Chave: Qualidade da água, PCH, Rio Jamari

\section{ABSTRACT}

With the construction of a SHP (small hydro) in the course of the Jamari River in the town of Monte Negro - RO becomes important monitoring of surface waters of the Rio Jamari to assess your current situation during the process of implementing the SHP to subsequently assess the impact caused the river by construction. The present paper has aimed at the evaluation of the water quality of the Rio Jamari, evaluating standards physico-chemical, microbiological, phytoplankton and ichthyoplankton, where after evaluating all the parameters generated up the WQI (Water Quality Index), which was between 68-79\%, assessed at four points in the course of the river, indicating a good quality water, within the parameters of evaluable IQA. The values 
Rev. Elet. em Gestão, Educação e Tecnologia Ambiental (e-ISSN: 2236-1170)

obtained were considered satisfactory in view of the Jamari River is a river that has suffered a lot from the process of deforestation in the region, deploying mines and plantations.

Keywords: Water quality, SHP and river Jamari

\section{INTRODUÇÃO}

A bacia amazônica representa a maior extensão de florestas tropicais da Terra, exercendo significativa influência no clima local e global, devido aos fluxos de energia e água na atmosfera. Presume-se que alteração dos ciclos da água, energia solar, carbono e nutrientes, resultantes da mudança no uso da terra na Amazônia possam provocar, consequências climáticas e ambientais em escalas local, regional e global (LUIZÃO, 2007).

A bacia hidrográfica do Rio Jamari está localizada em Rondônia, na região conhecida como Região do Vale do Jamari que engloba 09 cidades. Essa bacia vem sofrendo, nas últimas décadas, uma alteração nos seus recursos hídricos, tanto no aspecto qualitativo como no quantitativo, devido ao desmatamento, ocupação desordenada, queimadas, processos de garimpo, instalações de hidroelétricas no seu curso e a instalação de vários sítios e chácaras nas margens dos cursos de água.

A qualidade das águas superficiais de consumo humano no meio rural está diretamente relacionada à forma de ocupação do solo, à transformação de ecossistemas naturais equilibrados em áreas de lavouras, ao uso indiscriminado de agrotóxicos e fertilizantes e à falta de tratamento dos dejetos animais e humanos (ALMEIDA et al., 2001).

Uma Pequena Central Hidrelétrica $(\mathrm{PCH})$ é toda usina hidrelétrica de pequeno porte cuja capacidade instalada seja superior a $1 \mathrm{MW}$ e inferior a $30 \mathrm{MW}$. Além disso, a área do reservatório deve ser inferior a 3 km² (Resolução n.394 de 04/12/1998 da Agência Nacional de Energia Elétrica - ANEEL).

Sabendo-se da construção de uma PCH no município de Monte Negro no Rio Jamari, este trabalho tem por objetivo avaliar parâmetros limnológicos e da qualidade da água através de analises dos aspectos físico-químicos, microbiológicos, fitoplâncton e zooplâncton, durante o processo de instalação e antes do alagamento da área.

\section{MATERIAIS E MÉTODOS}

\section{Localização Geográfica}

A PCH (Figura 1) (latitude 1013'49,5”S e longitude 6314'01,5”W) situar-se-á no curso d'água do rio Jamari, município de Monte Negro, limita-se ao norte com Ariquemes, ao sul de Governador Jorge Teixeira, a leste de Cacaulândia e a oeste, Buritis e Campo Novo de Rondônia, tendo influencia de maneira direta e/ou indireta, em toda a região do Vale do Jamari, esta que outros trabalhos já demonstraram a preocupação de realização de acompanhamento da qualidade de suas águas (MENEGUETTI et al., 2011; MENEGUETTI et al., 2012). 
Rev. Elet. em Gestão, Educação e Tecnologia Ambiental (e-ISSN: 2236-1170)



Figura 1. Localização da PCH

\section{Qualidade das Águas Superficiais}

Foi realizada uma campanha de monitoramento da qualidade das águas superficiais do período de pré-enchimento, em quatro pontos na área de influência da $\mathrm{PCH}$, sendo escolhidos 02 a montante e 02 a jusante de onde será instalada a PCH. Foram coletas amostras para análise dos seguintes parâmetros apresentados na Tabela 01.

Tabela 01. Parâmetros de monitoramento

\begin{tabular}{cc}
\hline \multicolumn{2}{c}{ Parâmetros de monitoramento Físico-químico } \\
\hline Coliformes Termotolerantes & Turbidez \\
\hline Coliformes Totais & Cor \\
\hline Oxigênio Dissolvido: (OD) & Resíduo Total \\
\hline Demanda Biológica de Oxigênio (DBO) & Temperatura do ar \\
\hline Demanda Química de Oxigênio (DQO) & Temperatura da água \\
\hline pH & Óleos e graxas \\
\hline Nitrogênio Total & Condutividade Elétrica \\
\hline Fósforo Total & Sólidos dissolvidos Totais \\
\hline Parâmetros de monitoramento \\
\hline Fitoplâncton & Ictioplâncton
\end{tabular}




\section{Coleta das Amostras}

As amostras foram coletadas em quatro pontos distintos:

Ponto 1: Lat: $10^{\circ} 17^{\prime} 42.57^{\prime \prime S}$ Long: 6314'28.25"W (Figura 1)

Ponto 2: Lat: $10^{\circ} 18^{\prime} 2.04^{\prime \prime S}$ Long: 631' $20.91^{\prime \prime W}$ (Figura 2)

Ponto 3: Lat: $10^{\circ} 15^{\prime} 39.31^{\prime \prime S ~ L o n g: ~} 63^{\circ} 14^{\prime} 17.68^{\prime \prime W}$ (Figura 3)

Ponto 4: Lat: $10^{\circ} 13^{\prime} 41.22^{\prime \prime S}$ Long: $63^{\circ} 14^{\prime} 0.26^{\prime \prime} \mathrm{W}$ (Figura 4)
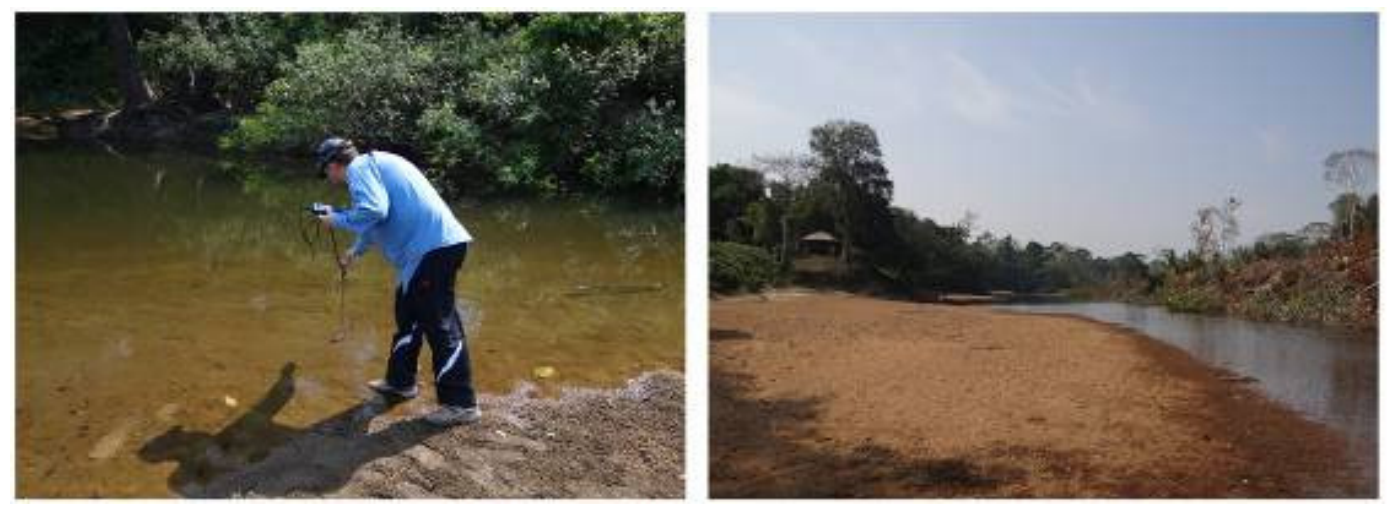

Figura 1. Primeiro ponto de coleta (montante).
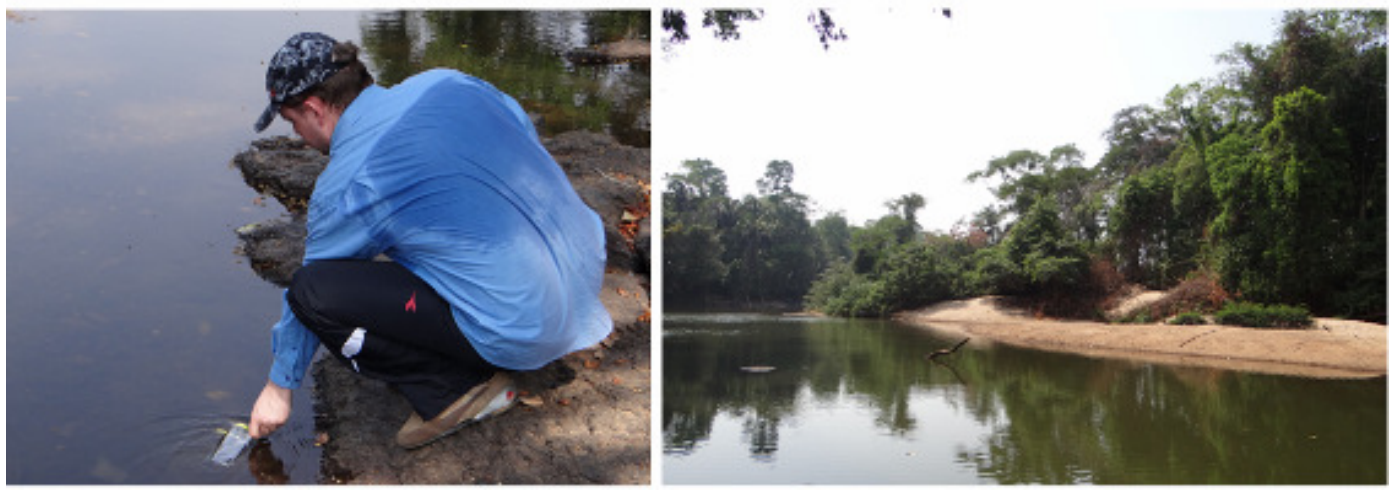

Figura 2. Segundo ponto de coleta (montante).
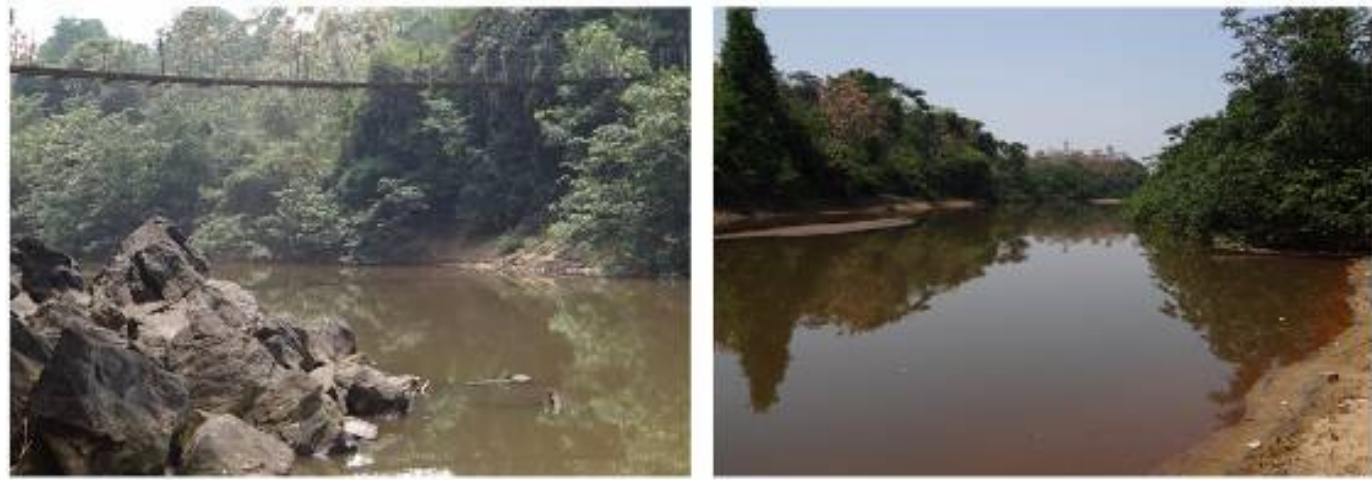

Figura 3. Terceiro ponto de coleta (jusante). 

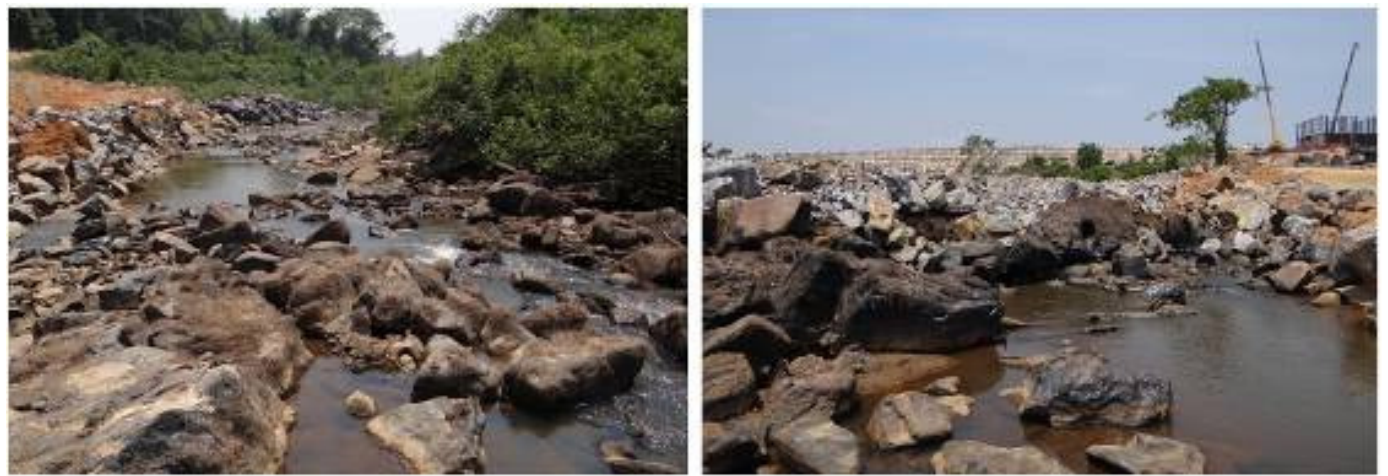

Figura 4. Quarto ponto de coleta (jusante).

\section{Análises Físico-químicas e Fitoplâncton e Zooplâncton}

A metodologia empregada nas análises físico-químicas foi baseada nos manuais Standard Methods for the Examination of Water and Wastewater, 19th Edition (APHA/AWWA/WEF, 1995) e da American Society for Testing of Materials (1992).

O Índice de Qualidade de Água (IQA) adotado foi o mesmo da $\operatorname{CETESB}(2003,2008)$ que é uma adaptação do índice de qualidade de água da National Sanitation Foundation (NSF), o qual é determinado pelo produtório ponderado dos valores obtidos para os parâmetros mencionados acima. A partir do cálculo efetuado, determina-se a qualidade das águas, que é indicada pelo IQA, o qual varia numa escala entre 0 a 100. De acordo com o resultado do IQA a qualidade da água é enquadrada em uma determinada categoria apresentada na Tabela 02.

Tabela 02. Valores dos padrões de IQA

\begin{tabular}{cc}
\hline Valor & Categoria \\
\hline $79<$ IQA $\leq 100$ & Ótima \\
\hline $51<$ IQA $\leq 79$ & Boa \\
\hline $36<$ IQA $\leq 51$ & Regular \\
\hline $19<$ IQA $\leq 36$ & Ruim \\
\hline IQA $\leq 19$ & Péssima
\end{tabular}

Os métodos de análise utilizados para Fitoplâncton e Zooplâncton foram:

As amostras foram concentradas em recipiente de vidro com capacidade de $100 \mathrm{~cm}^{3}$, e, com auxilio de uma pipeta foram retiradas várias alíquotas de $0,5 \mathrm{~cm}^{3}$ de cada amostra e formadas lâminas para microscopia. Estas foram observadas em um microscópio binocular, com aumento de 1600 vezes. Nas contagens, foi determinado o número de células por mililitro, registrando-se o número de espécie para cada divisão.

A identificação e enquadramento taxonômico do material foram realizados com auxilio de bibliografia especifica (SILVA et al., 1989; APRILE; MERA, 2007).

A determinação da densidade de organismos planctônicos expressa em $\mathrm{org} . / \mathrm{m}^{3}$ foi efetuada através da expressão (APHA,1995): 
Rev. Elet. em Gestão, Educação e Tecnologia Ambiental (e-ISSN: 2236-1170)

$$
\text { Org. } / m^{3}=D \times C^{\prime} / C^{\prime \prime} \times C^{\prime \prime \prime}
$$

$\mathrm{D}=\mathrm{n}$ o de organismos contados na alíquota analisada;

$\mathrm{C}^{\prime}$ = volume de concentração da amostra $(\mathrm{mL})$;

$$
\mathrm{C}^{\prime \prime}=\text { volume da alíquota analisada }(\mathrm{mL}) \text {; }
$$

\section{RESULTADOS E DISCUSSÕES}

Na tabela 03 estão apresentados os valores de análise dos parâmetros físico-químicos das amostras coletadas nos quatro pontos determinados.

\begin{tabular}{|c|c|c|c|c|c|}
\hline & \multirow[b]{2}{*}{ Limite* } & \multicolumn{2}{|c|}{ Montante } & \multicolumn{2}{|c|}{ jusante } \\
\hline & & Ponto 1 & Ponto 2 & Ponto 3 & Ponto 4 \\
\hline Temperatura do $\operatorname{ar}\left({ }^{\circ} \mathrm{C}\right)$ & -- & 37 & 36 & 37 & 37 \\
\hline Temperatura da água $\left({ }^{\circ} \mathrm{C}\right)$ & -- & 26 & 27 & 26 & 25 \\
\hline Alcalinidade $\left(\mathrm{mg}^{-1} \mathrm{~L}^{-1} \mathrm{CaCO}_{3}\right)$ & -- & 22 & 16 & 30 & 12 \\
\hline Nitrogênio total (mg. $\left.\mathrm{L}^{-1}\right)$ & 1,5 & 0,1821 & 0,859 & 0,242 & 0,1821 \\
\hline Fosforo Total (mg. $\mathrm{L}^{-1}$ ) & 0,1 & 0,2 & 0,23 & 0,18 & 0,09 \\
\hline Resíduos totais (mg. $\mathrm{L}^{-1}$ ) & 250 & 7 & 10,5 & 13,5 & 14 \\
\hline Cor (mg.L $\left.\mathrm{L}^{-1} \mathrm{Pt} / \mathrm{Co}\right)$ & 15 & 2,0 & 2,0 & 2,5 & 2,0 \\
\hline Dureza (mg. $\left.\mathrm{L}^{-1} \mathrm{CaCO}_{3}\right)$ & 500 & 14 & 16 & 50 & 76 \\
\hline Ferro (mg. $\left.\mathrm{L}^{-1} \mathrm{Fe}\right)$ & 0,3 & 0,20 & 0,25 & 0,15 & 0,35 \\
\hline Turbidez (mg. $\left.\mathrm{L}^{-1} \mathrm{NTU}\right)$ & 5,0 & 4,14 & 3,84 & 5,68 & 3,68 \\
\hline Oxigênio dissolvido (mg. $\mathrm{L}^{-1} \mathrm{O}_{2}$ ) & 6,0 & 12,5 & 20,0 & 14,0 & 13,0 \\
\hline $\mathrm{pH}$ (un. pH) & $6-9,5$ & 7,3 & 7,2 & 7,5 & 7,0 \\
\hline D.Q.O (mg. $\left.\mathrm{L}^{-1} \mathrm{O}_{2}\right)$ & 5,0 & 26,60 & 87,32 & 73,87 & 171,54 \\
\hline $\mathrm{DBO}\left(\mathrm{mg} \cdot \mathrm{L}^{-1}\right)$ & 5,0 & 2,5 & 2,1 & 2,8 & 1,9 \\
\hline Condutividade (Ohms) & -- & 50 & 75 & 64 & 76 \\
\hline Sólidos dissolvidos (mg. $\mathrm{L}^{-1}$ ) & 500 & 350 & 420 & 485 & 384 \\
\hline \multirow[t]{2}{*}{ Óleos e graxas (mg. $\left.\mathrm{L}^{-1}\right)$} & -- & 2,8 & 4,5 & 6,3 & 1,2 \\
\hline & Limites & Ponto 1 & Ponto 2 & Ponto 3 & Ponto 4 \\
\hline $\begin{array}{c}\text { Coliformes Totais (UFC/100 } \\
\mathrm{mL} \text { ) }\end{array}$ & Ausência & 2350 & 2458 & 2643 & 1987 \\
\hline $\begin{array}{c}\text { Coliformes Fecais (UFC/100 } \\
\mathrm{mL} \text { ) }\end{array}$ & Ausência & 2432 & 2541 & 2511 & 2152 \\
\hline $\begin{array}{l}\text { Indice de qualidade da água } \\
\text { (IQA) }\end{array}$ & Tabela 02 & $71 \%$ (boa) & $73 \%$ (boa) & $68 \%$ (boa) & $79 \%$ (boa) \\
\hline
\end{tabular}

Tabela 03. Valores de parâmetros físico-químicos

* Valores limites estabelecidos pela Resolução CONAMA 357/2005.

Os dados de temperatura da água apresentaram pequenas variações, cujos valores estiveram entre 25 e 27 으. Como tais medidas foram realizadas na superfície, foram influenciadas por fatores como latitude, altitude, época sazonal, período do dia, taxa de fluxo e profundidade. 0 teor de oxigênio dissolvido variou de 12,5 a 20,0 mg. $\mathrm{L}^{-1}$, o que indica que os valores estiveram acima daquilo que estabelece a Resolução CONAMA no 357/2005, que estabelece valores inferiores a $6 \mathrm{mg} \cdot \mathrm{L}^{-1}$.

Por outro lado, os valores de demanda bioquímica de oxigênio oscilaram entre 1,9 e 2,8 mg. ${ }^{-1}$, classificando-as dentro do estabelecido. Este padrão de classificação também foi seguido para os 
Rev. Elet. em Gestão, Educação e Tecnologia Ambiental (e-ISSN: 2236-1170)

valores de $\mathrm{pH}$, que mostraram variação entre 7 e 7,5, onde demonstraram tendência a este ser neutro.

As medidas de turbidez efetuadas em todos os pontos de coleta registraram variação de 3,68 a 5,68 dentro do estabelecido, exceto no ponto 03 que ficou um pouco acima do estabelecido. Em relação a esse parâmetro, é preciso considerar que as águas do Rio Jamari, como a maioria dos rios brasileiros, são naturalmente turvas em decorrência da natureza geológica de sua bacia de drenagem. Entretanto, há também contribuição das chuvas tropicais que carreiam componentes dos solos expostos, erodíveis ou agricultados, carreando partículas de argila, silte, fragmentos de rocha do solo para os ambientes aquáticos.

As quantidades totais de sólidos totais dissolvidos dos pontos de coleta localizados na região da montante e jusante não apresentaram padrão de variação entre montante e jusante, sendo que todos ficaram dentro dos padrões estabelecidos.

Para os coliformes termotolerantes, os valores encontrados em todos os pontos, foram maiores daquilo que está previsto na Resolução CONAMA n 274/2000, para o uso de recreação de contato primário e não foram obedecidos os padrões de qualidade de balneabilidade, previstos para os demais usos, onde não deveria ser excedido um limite de 200 coliformes termotolerantes por 100 mililitros.

Em relação aos nutrientes, que podem indicar a poluição orgânica, as águas foram avaliadas nas formas de fósforo total e nitrogênio total. O fósforo total indica diminuição progressiva em suas concentrações da montante em relação à jusante. O mesmo ocorreu com o nitrogênio total. $O$ valor de fósforo total, para ambiente lótico, estabelecido pela resolução CONAMA n ${ }^{0} 357 / 2005$ é de $0,1 \mathrm{mg} \cdot \mathrm{L}^{-1}$, e logo inferior ao observado nas análises, exceto no ponto 04 que ficou dentro dos padrões estabelecidos. Os resultados das análises físicas e químicas revelaram uma baixa variabilidade entre os pontos de coleta para a maioria das variáveis. As variáveis oxigênio dissolvido, coliformes fecais e fósforo total estiveram fora do limite estabelecido pela Resolução CONAMA n $357 / 2005$.

Após a realização dos testes físico-químicos, foi calculado o IQA e atribuído o conceito de qualidade de cada ponto de coleta. O resultado mostrou que todos os quatro pontos possuem uma qualidade de água boa, possuem um conceito de qualidade de água, a tabela 03 mostra os índices atribuídos a cada ponto e os respectivos conceitos da qualidade de água para o rio em cada ponto em relação à categoria referente a tabela 02.

\section{Resultados de Fitoplâncton}

\section{Dados qualitativos}

\section{Chlorophyta}

Classe Chlorococcales

Ordem Chlorococcales

Coelastrumsp

Oocystissp

Scenedesmussp

Ordem Trentepolhiales

Trentepolhiasp 
Rev. Elet. em Gestão, Educação e Tecnologia Ambiental (e-ISSN: 2236-1170)

\author{
Classe Zygnemaphyceae \\ Ordem Desmidiales \\ Actinotaenium sp1 \\ Actinotaenium sp2 \\ Actinotaenium sp3 \\ Closterium Sp1 \\ Closterium Sp2 \\ Closterium Sp3 \\ Closterium Sp4 \\ Cosmarium Sp1 \\ Cosmarium Sp2 \\ Cosmarium Sp3 \\ Euastrumsp \\ Netriumsp \\ Pleurotaeniumsp \\ Staurastrumsp
}

Ordem Zignemathales

Spirogyrasp

\title{
Euglenophyta
}

Classe Euglenophyceae

Ordem Euglenales
Euglena $s p$
Lepocinclissp
Phacussp
Trachelomonassp

\section{Chromophyta}

Classe bacillariophyceae

Ordem Eunotiales

$$
\begin{aligned}
& \text { Eunotia Sp1 } \\
& \text { Eunotia Sp2 } \\
& \text { Eunotia Sp3 }
\end{aligned}
$$

\section{Cyanophyta}

Classe Cyanophyceae

Ordem Nostocales

Ocilatoriasp

\section{Rhodophyta}

Classe Rhodophyceae

Ordem Acrochaetiales

Balliasp 
Rev. Elet. em Gestão, Educação e Tecnologia Ambiental (e-ISSN: 2236-1170)

\section{Dados Quantitativos}

Os dados qualitativos de Fitoplâncton são ilustrados nas (Tabelas 4, 5, 6, 7 e 8).

Tabela 4. Densidade total (org/mL) e densidade relativa (\%) de chlophyta

\begin{tabular}{ccc}
\hline Pontos de Amostra (Lat; Long) & \multicolumn{2}{c}{ Densidade } \\
\cline { 2 - 3 } & Org/mL & $\%$ \\
\hline Lat: $10^{\circ} 17^{\prime} 42.57^{\prime \prime} S$ Long: $63^{\circ} 14^{\prime} 28.25^{\prime \prime} \mathrm{W}$ & 348 & 30,27 \\
\hline Lat: $10^{\circ} 18^{\prime} 2.04^{\prime \prime} S$ Long: $63^{\circ} 14^{\prime} 20.91^{\prime \prime} \mathrm{W}$ & 326 & 28,45 \\
\hline Lat: $10^{\circ} 15^{\prime} 39.31^{\prime \prime} S$ Long: $63^{\circ} 14^{\prime} 17.68^{\prime \prime} \mathrm{W}$ & 280 & 24,43 \\
\hline Lat: $10^{\circ} 13^{\prime} 41.22^{\prime \prime} S$ Long: $63^{\circ} 14^{\prime} 0.26^{\prime \prime} \mathrm{W}$ & 192 & 16,75
\end{tabular}

Tabela 5. Densidade total (org/ $\mathrm{mL}$ ) e densidade relativa (\%) de Euglenophyta

\begin{tabular}{ccc}
\hline Pontos de Amostra (Lat; Long) & \multicolumn{2}{c}{ Densidade } \\
\cline { 2 - 3 } & Org/mL & $\%$ \\
\hline Lat: $10^{\circ} 17^{\prime} 42.57^{\prime \prime} S$ Long: $63^{\circ} 14^{\prime} 28.25^{\prime \prime} \mathrm{W}$ & 240 & 27,27 \\
\hline Lat: $10^{\circ} 18^{\prime} 2.04^{\prime \prime} S$ Long: $63^{\circ} 14^{\prime} 20.91^{\prime \prime} \mathrm{W}$ & 232 & 26,36 \\
\hline Lat: $10^{\circ} 15^{\prime} 39.31^{\prime \prime}$ S Long: $63^{\circ} 14^{\prime} 17.68^{\prime \prime} \mathrm{W}$ & 260 & 29,54 \\
\hline Lat: $10^{\circ} 13^{\prime} 41.22^{\prime \prime} S$ Long: $63^{\circ} 14^{\prime} 0.26^{\prime \prime} \mathrm{W}$ & 148 & 16,82
\end{tabular}

Tabela 6. Densidade total (org/mL) e densidade relativa (\%) de Chromophyta

\begin{tabular}{ccc}
\hline Pontos de Amostra (Lat; Long) & \multicolumn{2}{c}{ Densidade } \\
\cline { 2 - 3 } & Org/mL & $\%$ \\
\hline Lat: $10^{\circ} 17^{\prime} 42.57^{\prime \prime}$ S Long: $63^{\circ} 14^{\prime} 28.25^{\prime \prime} \mathrm{W}$ & 197 & 26,59 \\
\hline Lat: $10^{\circ} 18^{\prime} 2.04^{\prime \prime}$ S Long: $63^{\circ} 14^{\prime} 20.91^{\prime \prime} \mathrm{W}$ & 185 & 24,97 \\
\hline Lat: $10^{\circ} 15^{\prime} 39.31^{\prime \prime} S$ Long: $63^{\circ} 14^{\prime} 17.68^{\prime \prime} \mathrm{W}$ & 264 & 35,63 \\
\hline Lat: $10^{\circ} 13^{\prime} 41.22^{\prime \prime}$ S Long: $63^{\circ} 14^{\prime} 0.26^{\prime \prime} \mathrm{W}$ & 95 & 12,82
\end{tabular}

Tabela 7. Densidade total (org/mL) e densidade relativa (\%) de Cyanophyta

\begin{tabular}{ccc}
\hline Pontos de Amostra (Lat; Long) & \multicolumn{2}{c}{ Densidade } \\
\cline { 2 - 3 } & Org/mL & $\%$ \\
\hline Lat: $10^{\circ} 17^{\prime} 42.57^{\prime \prime} S$ Long: $63^{\circ} 14^{\prime} 28.25^{\prime \prime} \mathrm{W}$ & 38 & 28,57 \\
\hline Lat: $10^{\circ} 18^{\prime} 2.04^{\prime \prime} S$ Long: $63^{\circ} 14^{\prime} 20.91^{\prime \prime} \mathrm{W}$ & 32 & 24,06 \\
\hline Lat: $10^{\circ} 15^{\prime} 39.31^{\prime \prime} S$ Long: $63^{\circ} 14^{\prime} 17.68^{\prime \prime} \mathrm{W}$ & 39 & 29,32 \\
\hline Lat: $10^{\circ} 13^{\prime} 41.22^{\prime \prime} S$ Long: $63^{\circ} 14^{\prime} 0.26^{\prime \prime} \mathrm{W}$ & 24 & 18,04
\end{tabular}


Rev. Elet. em Gestão, Educação e Tecnologia Ambiental (e-ISSN: 2236-1170)

Tabela 8. Densidade total (org/mL) e densidade relativa (\%) de Rhodophyta

\begin{tabular}{ccc}
\hline Pontos de Amostra (Lat; Long) & \multicolumn{2}{c}{ Densidade } \\
\cline { 2 - 3 } & Org/mL & $\%$ \\
\hline Lat: $10^{\circ} 17^{\prime} 42.57^{\prime \prime} S$ Long: $63^{\circ} 14^{\prime} 28.25^{\prime \prime} \mathrm{W}$ & 28 & 27,45 \\
\hline Lat: $10^{\circ} 18^{\prime} 2.04^{\prime \prime} S$ Long: $63^{\circ} 14^{\prime} 20.91^{\prime \prime} \mathrm{W}$ & 26 & 25,49 \\
\hline Lat: $10^{\circ} 15^{\prime} 39.31^{\prime \prime} S$ Long: $63^{\circ} 14^{\prime} 17.68^{\prime \prime} \mathrm{W}$ & 34 & 33,33 \\
\hline Lat: $10^{\circ} 13^{\prime} 41.22^{\prime \prime} S$ Long: $63^{\circ} 14^{\prime} 0.26^{\prime \prime} \mathrm{W}$ & 14 & 13,72
\end{tabular}

O estudo de assembleias fitoplanctônicas é fundamentalmente importante quando se procura encontrar um padrão de influência sazonal ou de interferência antrópica.

Entre as Chlorophytas a ocorrência de espécies da ordem Desmidialesfoi a mais abundante, esta que é comum para a maioria de sistemas aquáticos amazônicos que apresentam condições limnológicas similares, sendo comum em rios com ou sem influência antrópica (FÖRSTER, 1963; FÖRSTER 1969).

As Bacillariophyceae, com predominância do gênero Eunotia, também representam um padrão dos ambientes oligotróficos de águas claras ou pretas onstituídas pelos rios que adentram na floresta (MERA, 1995).

A não dominância das Cyanophyceae e Euglenophyceae, utilizadas como indicadores de ambientes eutrofizados, e os baixos teores de condutividade e nutrientes inorgânicos no sistema, refletem diretamente na instabilidade da qualidade da água do rio Jamari, porém tem a necessidade de um acompanhamento sazonal para averiguar a variação das mesmas e ter um parâmetro completo do equilíbrio da dinâmica e estrutura das populações para melhores conclusões, principalmente durante a influência antrópica ocasionada pelas inundações das PCHs (APRILE; MERA, 2007).

A baixa ocorrência de Rhodophyta era esperada devido as poucas espécies ocorrentes em água doce, porém é necessário o acompanhamento da proliferação das mesmas durante as estações e alagamento ocasionado pelas $\mathrm{PCH}$ s para melhores conclusões.

\title{
Resultados do Zooplâncton Dados qualitativos \\ Copépodes
}

Ordem: Cyclopoidasp

Ordem: Calanoidasp

Ordem: Copepoditossp

\section{Cladóceros}

\author{
Bosminopsissp \\ Bosminasp \\ Moina sp \\ Diaphanosomasp
}


Rev. Elet. em Gestão, Educação e Tecnologia Ambiental (e-ISSN: 2236-1170)

\section{Rotíferos}

\section{Lecanesp \\ Keratellasp \\ Brachionussp}

\section{Dados Quantitativos}

Os dados qualitativos de Zooplâncton são ilustrados nas (Tabelas 9, 10 e 11).

Tabela 9. Densidade total (org/mL) e densidade relativa (\%) de Copépodes

\begin{tabular}{ccc}
\hline Pontos de Amostra (Lat; Long) & \multicolumn{2}{c}{ Densidade } \\
\cline { 2 - 3 } & Org/mL & $\%$ \\
\hline Lat: $10^{\circ} 17^{\prime} 42.57^{\prime \prime}$ S Long: $63^{\circ} 14^{\prime} 28.25^{\prime \prime} \mathrm{W}$ & 24 & 30,00 \\
\hline Lat: $10^{\circ} 18^{\prime} 2.04^{\prime \prime} S$ Long: $63^{\circ} 14^{\prime} 20.91^{\prime \prime} \mathrm{W}$ & 26 & 32,50 \\
\hline Lat: $10^{\circ} 15^{\prime} 39.31^{\prime \prime} S$ Long: $63^{\circ} 14^{\prime} 17.68^{\prime \prime} \mathrm{W}$ & 19 & 23,75 \\
\hline Lat: $10^{\circ} 13^{\prime} 41.22^{\prime \prime} S$ Long: $63^{\circ} 14^{\prime} 0.26^{\prime \prime} \mathrm{W}$ & 11 & 13,75
\end{tabular}

Tabela 10. Densidade total (org/mL) e densidade relativa (\%) de Cladóceros

\begin{tabular}{ccc}
\hline Pontos de Amostra (Lat; Long) & \multicolumn{2}{c}{ Densidade } \\
\cline { 2 - 3 } & Org/mL & $\%$ \\
\hline Lat: $10^{\circ} 17^{\prime} 42.57^{\prime \prime} S$ Long: $63^{\circ} 14^{\prime} 28.25 " \mathrm{~W}$ & 28 & 33,73 \\
\hline Lat: $10^{\circ} 18^{\prime} 2.04^{\prime \prime} S$ Long: $63^{\circ} 14^{\prime} 20.91^{\prime \prime} \mathrm{W}$ & 25 & 30,12 \\
\hline Lat: $10^{\circ} 15^{\prime} 39.31^{\prime \prime} S$ Long: $63^{\circ} 14^{\prime} 17.68^{\prime \prime} \mathrm{W}$ & 21 & 25,30 \\
\hline Lat: $10^{\circ} 13^{\prime} 41.22^{\prime \prime} S$ Long: $63^{\circ} 14^{\prime} 0.26 " \mathrm{~W}$ & 9 & 10,84
\end{tabular}

Tabela 11. Densidade total (org/mL) e densidade relativa (\%) de Rotíferos

\begin{tabular}{ccc}
\hline Pontos de Amostra (Lat; Long) & \multicolumn{2}{c}{ Densidade } \\
\cline { 2 - 3 } & Org/mL & $\%$ \\
\hline Lat: $10^{\circ} 17^{\prime} 42.57^{\prime \prime} S$ Long: $63^{\circ} 14^{\prime} 28.25^{\prime \prime} \mathrm{W}$ & 11 & 35,38 \\
\hline Lat: $10^{\circ} 18^{\prime} 2.04^{\prime \prime}$ S Long: $63^{\circ} 14^{\prime} 20.91^{\prime \prime} \mathrm{W}$ & 8 & 25,81 \\
\hline Lat: $10^{\circ} 15^{\prime} 39.31^{\prime \prime} S$ Long: $63^{\circ} 14^{\prime} 17.68^{\prime \prime} \mathrm{W}$ & 8 & 25,81 \\
\hline Lat: $10^{\circ} 13^{\prime} 41.22^{\prime \prime}$ S Long: $63^{\circ} 14^{\prime} 0.26^{\prime \prime} \mathrm{W}$ & 4 & 12,90
\end{tabular}

A ocorrência de rotíferos deve-se provavelmente ás condições propícias de alimentos somadas ás características peculiares desses organismos quanto à reprodução, ocorrendo durante a seca quando ha redução da concentração de oxigênio na água.

Os cladóceros são zooplânctons com tamanho elevado, sua grande ocorrência pode se da por dois fatores: 10 quando o mesmo utiliza macrófitas como refúgio contra peixes predadores, ou quando a quando ocorre a diminuição da quantidade de peixes ocorrentes no rio, havendo assim a proliferação desses organismos. 
Rev. Elet. em Gestão, Educação e Tecnologia Ambiental (e-ISSN: 2236-1170)

Acredita-se que os copépodes são o grupo de metazários mais numeroso do mundo. Formam elo importante no fluxo de transferência de energia dos ecossistemas aquáticos. Larvas de muitas espécies de peixe e mesmo adultos de algumas outras alimentam-se de copépodes, podendo também ser parasitados pelos mesmos. Eles podem suportar grades variações de temperatura da água $\left(10\right.$ a $\left.30^{\circ} \mathrm{C}\right)$, sendo tolerantes também a falta de alimentos, devido a capacidade de armazenamento de energia, os mesmos conseguem sobreviver em águas com concentração alta de poluição.

\section{CONCLUSÕES}

Tendo em vista que a grande maioria dos parâmetros avaliados encontraram-se dentro dos padrões estabelecidos pela resolução do CONAMA 357/05 para águas de classe 2, mesmo sob pressões antrópicas, sendo que também não foi observado nenhum local eutrofizado, ou seja, com níveis de nutrientes elevados, pode-se concluir que a água está com uma boa qualidade e dentro das normalidades.

É importante lembrar que a comunidade regional e local está em crescimento/desenvolvimento em decorrência da expansão agrícola, pecuária e implantação de indústrias, $\mathrm{PCH}$ 's, e estes fatores levam a exploração de forma inadequada do meio ambiente, o que pode vir a ocasionar impactos futuros na qualidade da água.

\section{REFERÊNCIAS}

ALMEIDA, S. G. et al. Crise socioambiental e conversão ecológica da agricultura brasileira: subsídios à formação de diretrizes ambientais para o desenvolvimento agrícola. Rio de Janeiro: AS-PTA, 2001. 122p.

AMERICAN PLUBLIC HEALT ASSOCIATION. Standard methods for the examination of water and wastewater, 19th edition, Washington.APHA, 1995.

American Society for Testing of Materials.Annual Book of ASTM Standards, ASTM, 1992.

APRILE, F.M; MERA, P.A.S. Fitoplâncton e Fitoperifíton de um rio de águas pretas sa Amazônia periférica do norte, Brasil. Braz. J. Aquat. Sci. Technol, v.11, n.2, p.1-14, 2007.

CETESB. Relatório de qualidade das águas interiores do Estado de São Paulo. São Paulo, 2003.

CETESB. Relatório de qualidade das águas interiores do Estado de São Paulo. São Paulo, 2008.

Conselho Nacional do Meio Ambiente (CONAMA). Resolução no 357, de 17 de Março de 2005.

LUIZÃO, F. J. Ciclos de nutrientes na Amazônia: respostas às mudanças ambientais e climáticas. Ciência e Cultura, v.59, n.3, p.31-36, 2007. 
FÖRSTER, K. Desmidiaceen aus Brasilien, 1: Nord Brasilien. Revue Algologique, v.7, n.1, p.38-92, 1963.

FÖRSTER, K. Amasonische Desmidiaceen, 1: Areal Santarém. Amazoniana, v.2, n.12, p.5-23I, 1969.

MERA, P.A.S. Características Ecológicas da Desmidioflórula de uma Região Hidrográfica do Sistema Trombetas, Estado do Pará, Brasil. Tese de Doutorado, INPA/FUA, Manaus, 1995, 237p.

MENEGUETTI, D.U.O; SILVA, F.C; ZAN, R.A; RAMOS, L.J. Adaptation of the micronucleus technique in Allium cepa, for mutagenicity analysis of the Jamari river valley, western Amazon, Brazil. J Environment Analytic Toxicol v.2, n.2, p.127, 2012.

MENEGUETTI, D.U.O; SILVA, F.C; ZAN, R.A; POLETTO, P.O; RAMOS, L.J. Adaptação da técnica de micronúcleo em Allium cepa, para futuras análises de mutagenicidade dos rios da região do Vale do Jamari, Rondônia, Amazônia ocidental. Revista Pesquisa \& Criação, v.10, n.2, p.181-187, 2011.

SILVA, E.N.S; ROBERTSON, B.A; REID, J.L.W; HARDY, E.R.. Atlas de copépodos planctônicos, Calanoida e Cyc1opoida (Crustacea), da Amazônia Brasileira. I. Represa de Curuá-Una, Pará. Rev bras. Zool, v.6, n.4, p.725-758, 1989. 